$1-1-1996$

\title{
Dielectric Screening In A Plasma: Some Rigorous Results
}

Bhimsen K. Shivamoggi

University of Central Florida

Find similar works at: https://stars.library.ucf.edu/facultybib1990 University of Central Florida Libraries http://library.ucf.edu

This Article is brought to you for free and open access by the Faculty Bibliography at STARS. It has been accepted for inclusion in Faculty Bibliography 1990s by an authorized administrator of STARS. For more information, please contactSTARS@ucf.edu.

\section{Recommended Citation}

Shivamoggi, Bhimsen K., "Dielectric Screening In A Plasma: Some Rigorous Results" (1996). Faculty Bibliography 1990s. 1755.

https://stars.library.ucf.edu/facultybib1990/1755

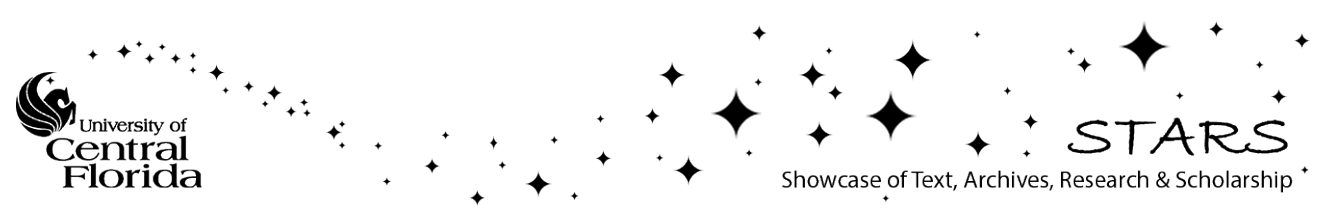




\section{Dielectric screening in a plasma: Some rigorous results}

Cite as: Journal of Mathematical Physics 37, 254 (1996); https://doi.org/10.1063/1.531389

Submitted: 31 July 1995 . Accepted: 19 September 1995. Published Online: 04 June 1998

Bhimsen K. Shivamoggi

\section{AVS Quantum Science}

A high impact interdisciplinary journal for ALL quantum science 


\title{
Dielectric screening in a plasma: Some rigorous results
}

\author{
Bhimsen K. Shivamoggi ${ }^{\mathrm{a})}$ \\ Technische Hochschule Darmstadt, Germany
}

(Received 31 July 1995; accepted for publication 19 September 1995)

In this article, we give some rigorous results about the properties of the solution of Poisson's equation describing dielectric screening by electrons trapped and/or untrapped in the Coulomb field of the test charge. (C) 1996 American Institute of Physics. [S0022-2488(96)02501-6]

\section{INTRODUCTION}

Introduction of a test charge $q$ (say, at $\mathbf{r}=\mathbf{0}$ ) polarizes the plasma and produces a shielding cloud around the charge. If the plasma were cold and there were no thermal agitation, the shielding would be perfect and the potential of the test charge would drop to zero outside the cloud. The test charge would be electrically invisible there! If the plasma were not cold, however, there would be a few particles at the edge of the cloud which would have enough thermal energy to escape from the cloud so that the shielding will not be complete.

The effective potential field $\phi(\mathbf{r})$ around the test charge $q$ is calculated by taking into account the dielectric screening produced by other charged particles. Poisson's equation gives

$$
\nabla^{2} \phi=-4 \pi\left(n_{i}-n_{e}\right)-4 \pi q \delta(\mathbf{r}),
$$

where $n$ is the number density of the particles and the subscripts $i$ and $e$ denote the ions and the electrons, respectively. The ions are assumed not to participate in the shielding process and to form only a uniform neutralizing background, so

$$
n_{i}=n_{0} .
$$

The full (trapped plus untrapped) electrons subjected to the Coulomb field of the test charge are assumed to be distributed in space according to the Boltzmann distribution

$$
n_{e}(\mathbf{r})=n_{0} e^{(e \phi) / k T_{e}},
$$

$T$ being the temperature.

If the plasma is weakly coupled, the coupling parameter $\Gamma$ satisfies

$$
\Gamma=\frac{\text { mean Coulomb interaction energy }}{\text { thermal energy }} \sim\left|\frac{e \phi}{K T_{e}}\right| \ll 1 .
$$

Equation (4), in conjunction with Eqs. (2) and (3), leads to a linearization of Eq. (1):

$$
\nabla^{2} \phi_{\operatorname{lin}}=\lambda_{D}^{-2} \phi_{\operatorname{lin}}-4 \pi q \delta(\mathbf{r})
$$

with the well-known solution ${ }^{1}$

$$
\phi_{\operatorname{lin}}=\frac{q}{r} e^{-(r) / \lambda_{D}},
$$

\footnotetext{
${ }^{a)}$ Permanent address: University of Central Florida, Orlando, Florida.
} 
where $\lambda_{D}$ is the Debye length

$$
\lambda_{D}=\sqrt{\frac{K T_{e}}{4 \pi n_{0} e^{2}}}
$$

On the other hand, the electrons untrapped by the Coulomb field of the test charge are distributed according to the Maxwell-Boltzmann distribution

$$
f_{e}^{(u)}(\mathbf{r}, \mathbf{v})=\left\{\begin{array}{l}
n_{0}\left(\frac{m_{e}}{2 \pi K T_{e}}\right)^{3 / 2} \exp \left[-\left(\frac{m_{e} \nu^{2}}{2}-e \phi\right) \frac{1}{K T_{e}}\right], \quad \frac{m_{e} \nu^{2}}{2}>|e \phi| \\
0, \quad \frac{m_{e} \nu^{2}}{2}<|e \phi|
\end{array} .\right.
$$

The number density of the untrapped electrons is then given by

$$
n_{e}^{(u)}(\mathbf{r})=\int_{0}^{\infty} f_{e}^{(u)}(\mathbf{r}, \mathbf{v}) 4 \pi \nu^{2} d \nu=n_{0}\left\{e^{(e \phi) / K T_{e}}\left[1-e r f\left(\sqrt{\frac{e \phi}{K T_{e}}}\right)\right]+\frac{2}{\sqrt{\pi}} \sqrt{\frac{e \phi}{K T_{e}}}\right\} .
$$

The number of the trapped electrons, from Eqs. (3) and (8), is thus given by

$$
n_{e}^{(t)}(\mathbf{r})=n_{e}(\mathbf{r})-n_{e}^{(u)}(\mathbf{r})=n_{0}\left[e^{(e \phi) / K T_{e}} e r f\left(\sqrt{\frac{e \phi}{K T_{e}}}\right)-\frac{2}{\sqrt{\pi}} \sqrt{\frac{e \phi}{K T_{e}}}\right] .
$$

In this paper, we propose to give some rigorous results about the properties of the solution of Eq. (1) in conjunction with Eq. (3), (8), or (9).

\section{SCREENING BY THE FULL ELECTRONS}

Let us nondimensionalize according to

$$
\hat{\phi}=\frac{e \phi}{K T_{e}}, \quad \hat{r}=\frac{r}{\lambda_{D}},
$$

and drop the hats. Equation (1), on using Eqs. (2) and (3), then becomes

$$
(r \phi)^{\prime \prime}=r\left(e^{\phi}-1\right)-r q \delta(\mathbf{r})
$$

where primes denote differentiation with respect to $r$.

Lampert and Crandall ${ }^{2}$ rigorously proved that the solution of Eq. (11) is monotonically decreasing. The occurrence of dielectric screening would indicate that this result can be sharpened further, as given below.

Theorem 1: Let $\phi$ be a solution of

$$
(r \phi)^{\prime \prime}=r\left(e^{\phi}-1\right)-r q \delta(\mathbf{r}) .
$$

Then $\phi$ decreases more rapidly than the shieldless decay law $1 / r, \forall r$.

Proof: The proof is adapted from that given by Lampert and Crandall. ${ }^{2}$

Let $\phi>0, \forall r$. Upon integrating Eq. (11) once over $0<r_{1} \leqslant r \leqslant r_{2}<\infty$, we obtain

$$
(r \phi)_{2}^{\prime}-(r \phi)_{1}^{\prime}=\int_{r_{1}}^{r_{2}} r\left(e^{\phi}-1\right) d r .
$$




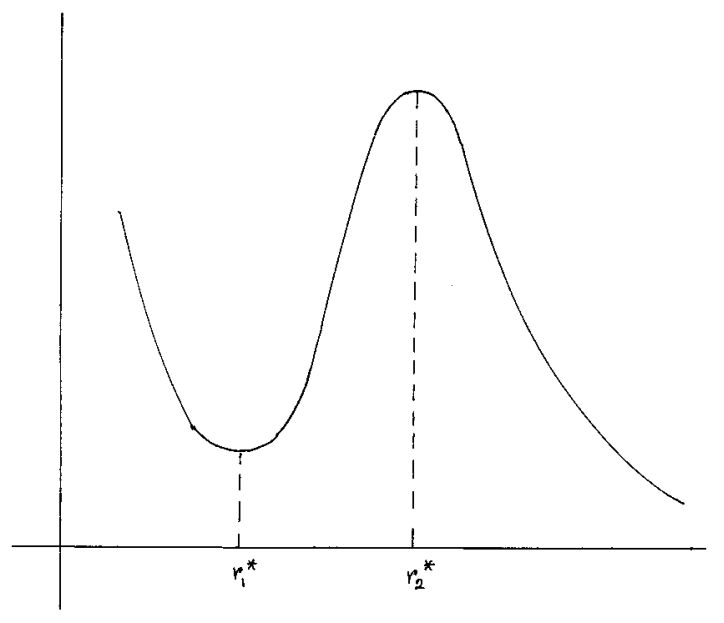

FIG. 1. $r \phi$ vs $r$.

If we now suppose that $(r \phi)$ does not decrease monotonically with $r$, then $(r \phi)$ must have a minimum for $r=r_{1}^{*}<\infty$. However, since

$$
e^{\phi}-1 \approx \phi, \quad \phi \ll 1
$$

$\phi$ must eventually decrease more rapidly than $1 / r$; so it is clear that $(r \phi)$ must have a maximum for $r=r_{2}^{*}$ with $r_{1}^{*}<r_{2}^{*}<\infty$ (see Fig. 1).

Taking $r_{1}=r_{1}^{*}$ and $r_{2}=r_{2}^{*}$, Eq. (12) gives

$$
0=\int_{r_{1}^{*}}^{r_{2}^{*}} r\left(e^{\phi}-1\right) d r
$$

which is, of course, impossible since $\phi>0, \forall r$.

Now, according to Eq. (13), the dielectric screening by the full electrons resembles, in the far field $(r \gg 1)$, a linearized screening. (Physically this is appreciable because, according to Eqs. (8) and (9), the untrapped electrons outnumber the trapped electrons, for $r \gg 1$.) However, the numerical work of Lampert and Crandall ${ }^{2}$ as well as that of $\mathrm{Mak}^{3}$ indicated that the linearization condition (4) is unnecessarily stringent and that the exact solution of Eq. (11) remains close to the linearized solution (6) well after the linearization condition (4) is violated. Actually, the exact solution is bounded from below by the linearized solution (6), as shown below.

Lemma 1: The function

$$
f(\phi)=e^{\phi}-1-\phi, \quad \phi>0
$$

is positive and monotonically increasing (see Fig. 2).

Theorem 2: Let $\phi$ be a solution of

$$
(r \phi)^{\prime \prime}=r\left(e^{\phi}-1\right)-r q \delta(\mathbf{r}) .
$$

Then, $\phi$ is bounded from below, $\forall r$, by the linearized solution 


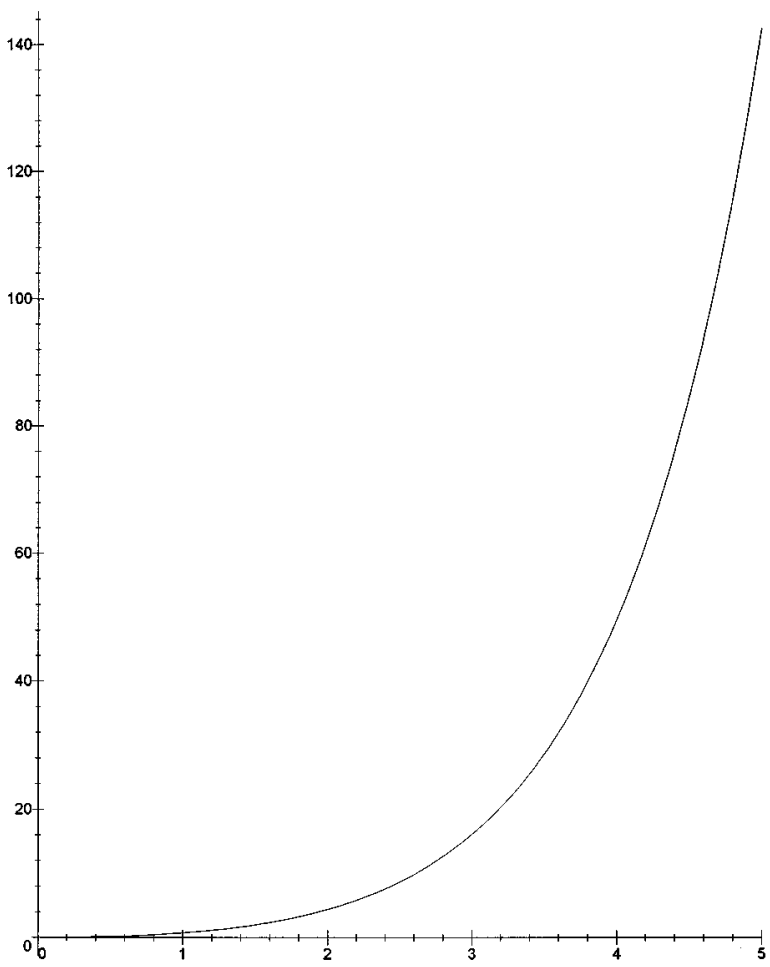

FIG. 2. $f(\phi)$ vs $\phi$.

$$
\phi_{\operatorname{lin}}=\frac{q}{r} e^{-\mathbf{r}}
$$

Proof: The exact solution of Eq. (11) can be written formally as

$$
(r \phi)=\int_{r}^{\infty}\left(r^{\prime}-r\right) r^{\prime}\left[\phi\left(r^{\prime}\right)+\left\{e^{\phi\left(r^{\prime}\right)}-1-\phi\left(r^{\prime}\right)\right\}-q \delta\left(\mathbf{r}^{\prime}\right)\right] d r
$$

from which

$$
(r \phi)=q e^{-r}+\int_{r}^{\infty}\left(r^{\prime}-r\right) r^{\prime}\left[e^{\phi\left(r^{\prime}\right)}-1-\phi\left(r^{\prime}\right)\right] d r^{\prime} .
$$

Using Lemma 1, Eq. (16) then implies that

$$
(r \phi) \geqslant q e^{-r}, \forall r .
$$

According to Theorem 2, the exact screening of the test change is weaker than linearized screening for the full electrons. 


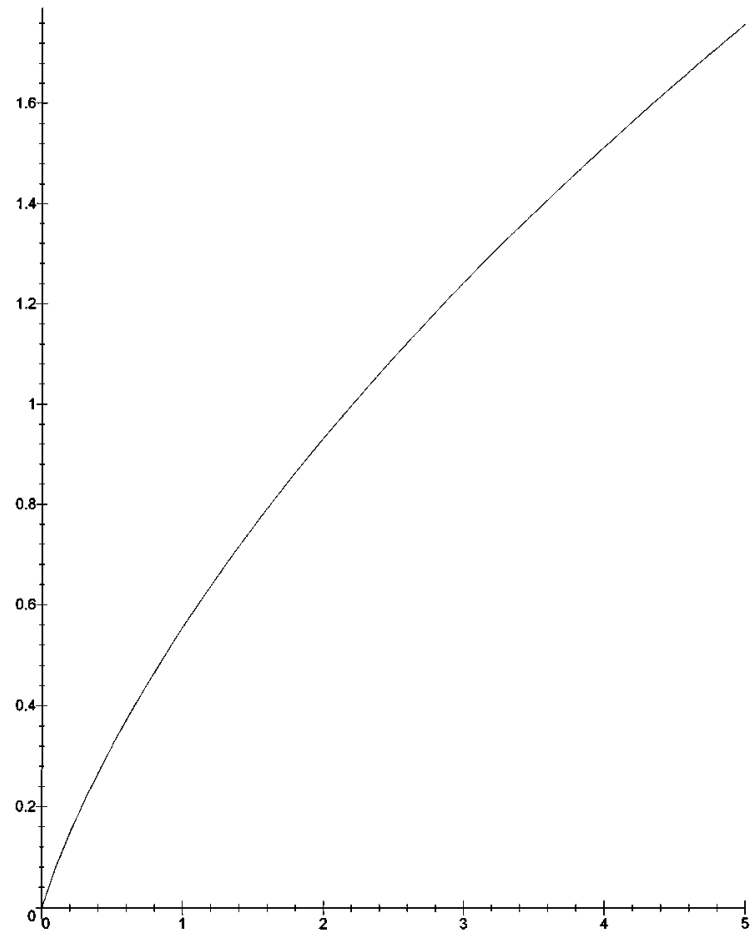

FIG. 3. $F(\phi)$ vs $\phi$.

\section{SCREENING BY UNTRAPPED ELECTRONS}

On nondimensionalizing according to Eq. (10), and using Eqs. (2) and (8), Eq. (1) gives for the untrapped-electron screening

$$
(r \phi)^{\prime \prime}=r\left[e^{\phi}\{1-\operatorname{erf}(\sqrt{\phi})\}+\frac{2}{\sqrt{\pi}} \sqrt{\phi}-1\right]-r q \delta(\mathbf{r}) .
$$

The occurrence of dielectric screening again implies that the solution of Eq. (17) decreases more rapidly than the shieldless decay law $1 / r, \forall r$, as deduced below.

Lemma 2: The function

$$
F(\phi)=e^{\phi}[1-e r f(\sqrt{\phi})]+\frac{2}{\sqrt{\pi}} \sqrt{\phi}-1
$$

is positive and monotonically increasing (see Fig. 3).

Theorem 3: Let $\phi$ be a solution of

$$
(r \phi)^{\prime \prime}=r\left[e^{\phi}\{1-\operatorname{erf}(\sqrt{\phi})\}+\frac{2}{\sqrt{\pi}} \sqrt{\phi}-1\right]-r q \delta(\mathbf{r}) .
$$

Then, $\phi$ decreases more rapidly than $1 / r, \forall r$.

Proof: The proof is similar to that for Theorem 1. 
Let $\phi>0, \forall r$. Upon integrating Eq. (17) once over $0<r_{1} \leqslant r \leqslant r_{2}<\infty$, we obtain

$$
(r \phi)_{2}^{\prime}-(r \phi)_{1}^{\prime}=\int_{r_{1}}^{r_{2}}\left[e^{\phi}\{1-e r f(\sqrt{\phi})\}+\frac{2}{\sqrt{\pi}} \sqrt{\phi}-1\right] d r .
$$

If we now suppose that $(r \phi)$ does not decrease monotonically with $r$, then $(r \phi)$ must have a minimum for $r=r_{1}^{*}<\infty$. However, since

$$
e^{\phi}[1-e r f(\sqrt{\phi})]+\frac{2}{\sqrt{\pi}} \sqrt{\phi}-1 \approx \phi, \quad \phi \ll 1
$$

we have from Eq. (17) that

$$
\phi_{\operatorname{lin}} \sim \frac{q}{r} e^{-r}, \quad r \gg 1
$$

showing that $\phi$ eventually decreases more rapidly than $1 / r$. Therefore, $(r \phi)$ must have a maximum for $r=r_{2}^{*}$ with $r_{1}^{*}<r_{2}^{*}<\infty$.

Taking $r_{1}=r_{1}^{*}$ and $r_{2}=r_{2}^{*}$ Eq. (19) then gives

$$
0=\int_{r_{1}^{*}}^{r_{2}^{*}} r\left[e^{\phi}\{1-\operatorname{erf}(\sqrt{\phi})\}+\frac{2}{\sqrt{\pi}} \sqrt{\phi}-1\right] d r
$$

which, from Lemma 2, is impossible.

Therefore, $(r \phi)$ must decrease monotonically, $\forall r$.

Now, in view of Eq. (20), the dielectric screening by untrapped electrons resembles, in the far field $(r \gg 1)$, a linearized screening. However, the exact screening of the test charge by the untrapped electrons turns out to be stronger than linearized screening, as deduced below.

Lemma 3: The function

$$
G(\phi)=e^{\phi}[1-\operatorname{erf}(\sqrt{\phi})]+\frac{2}{\sqrt{\pi}} \sqrt{\phi}-1-\phi
$$

is negative and monotonically decreasing (see Fig. 4).

Theorem 4: Let $\phi$ be a solution of

$$
(r \phi)^{\prime \prime}=r\left[e^{\phi}\{1-\operatorname{erf}(\sqrt{\phi})\}+\frac{2}{\sqrt{\pi}} \sqrt{\phi}-1\right]-r q \delta(\mathbf{r}) .
$$

Then, $\phi$ is bounded from above, $\forall r$, by the linearized solution

$$
\phi_{\operatorname{lin}}=\frac{q}{r} e^{-r}
$$

Proof: The exact solution of Eq. (17) can be written formally as

$$
\begin{aligned}
(r \phi)= & \int_{r}^{\infty}\left(r^{\prime}-r\right) r^{\prime}\left[\phi\left(r^{\prime}\right)+\left\{e^{\phi\left(r^{\prime}\right)}\left[1-e r f\left(\sqrt{\phi\left(r^{\prime}\right)}\right)\right]+\frac{2}{\sqrt{\pi}} \sqrt{\phi\left(r^{\prime}\right)}-1-\phi\left(r^{\prime}\right)\right\}\right. \\
& \left.-q \delta\left(\mathbf{r}^{\prime}\right)\right] d r^{\prime}
\end{aligned}
$$




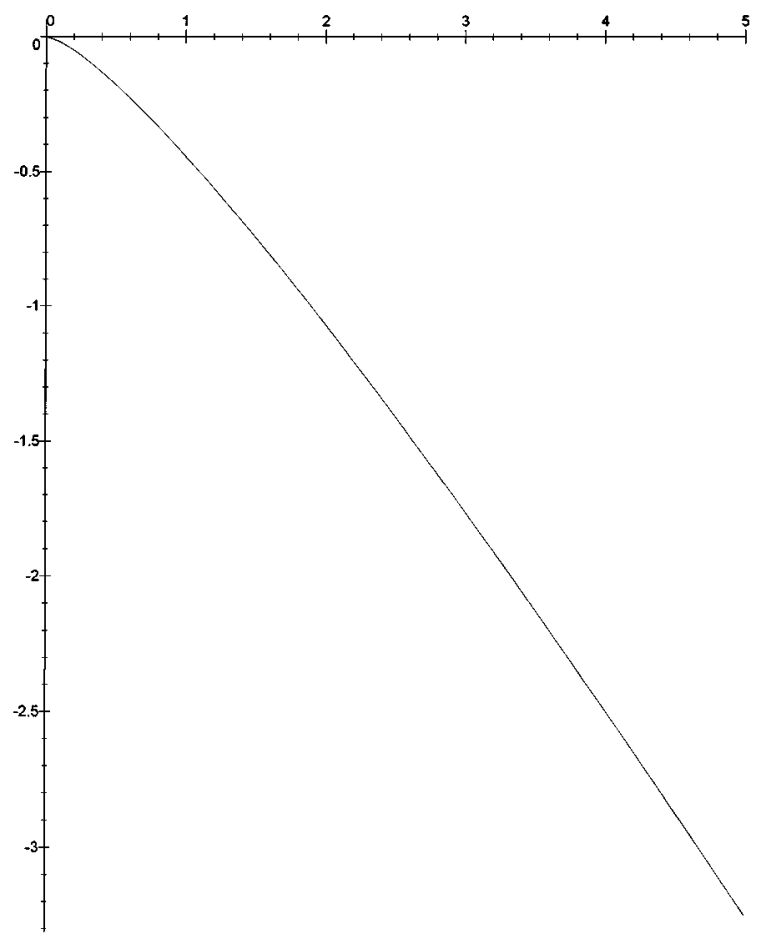

FIG. 4. $G(\phi)$ vs $\phi$.

from which

$$
(r \phi)=q e^{-r}+\int_{r}^{\infty}\left(r^{\prime}-r\right) r^{\prime}\left\{e^{\phi\left(r^{\prime}\right)}\left[1-e r f\left(\sqrt{\phi\left(r^{\prime}\right)}\right)\right]+\frac{2}{\sqrt{\pi}} \sqrt{\phi\left(r^{\prime}\right)}-1-\phi\left(r^{\prime}\right)\right\} d r^{\prime} .
$$

Using Lemma 3, Eq. (24) then implies that

$$
(r \phi) \leqslant q e^{-r}, \quad \forall r .
$$

According to Theorem 4, the exact screening of the test change is stronger than linearized screening for the untrapped electrons.

\section{SCREENING BY TRAPPED ELECTRONS}

Nondimensionalizing according to Eq. (10), and using Eqs. (2) and (9), Eq. (1) gives for the trapped-electron screening,

$$
(r \phi)^{\prime \prime}=r\left[e^{\phi} \operatorname{erf}(\sqrt{\phi})-\frac{2}{\sqrt{\pi}} \sqrt{\phi}\right]-r q \delta(\mathbf{r}) .
$$

The solution of Eq. (2) decreases again more rapidly than the shieldless decay law $1 / r, \forall r$, as deduced below. 


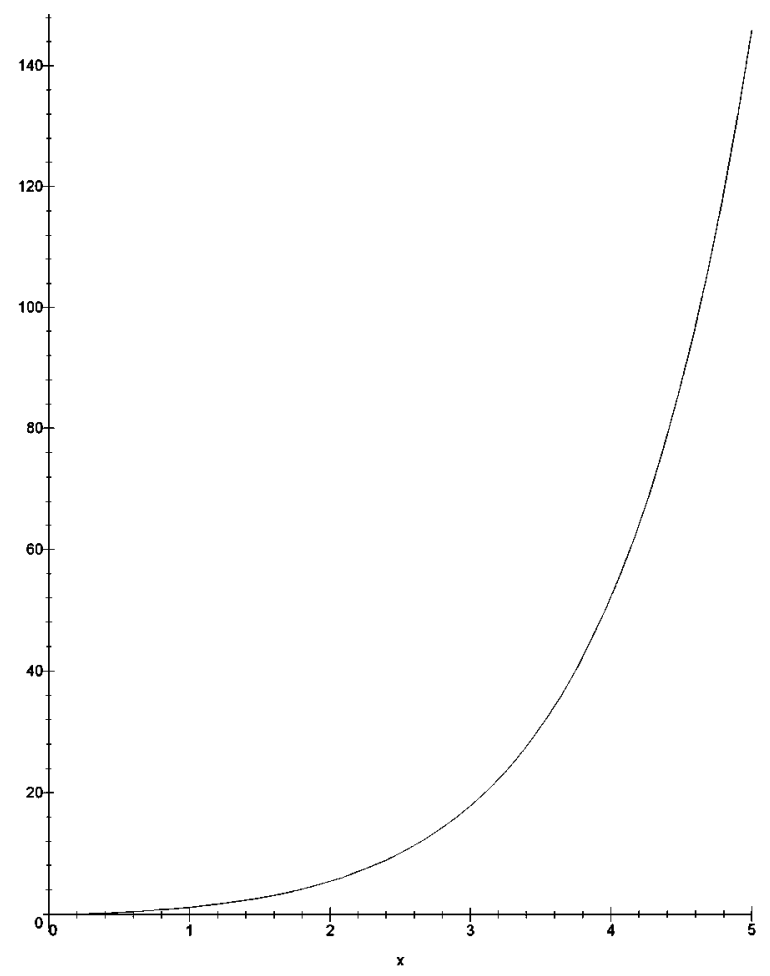

FIG. 5. $H(\phi)$ vs $\phi$.

Lemma 4: The function

$$
H(\phi)=e^{\phi} \operatorname{erf}(\sqrt{\phi})-\frac{2}{\sqrt{\pi}} \sqrt{\phi}
$$

is positive and monotonically increasing (see Fig. 5).

Theorem 5: Let $\phi$ be a solution of

$$
(r \phi)^{\prime \prime}=r\left[e^{\phi} \operatorname{erf}(\sqrt{\phi})-\frac{2}{\sqrt{\pi}} \sqrt{\phi}\right]-r q \delta(\mathbf{r}) .
$$

Then, $\phi$ decreases more rapidly than $1 / r, \forall r$.

Proof: The proof is entirely similar to that of Theorem 3 and uses Lemma 4. Now, since

$$
e^{\phi} \operatorname{erf}(\sqrt{\phi})-\frac{2}{\sqrt{\pi}} \sqrt{\phi} \approx \frac{2}{\sqrt{\pi}} \phi^{3 / 2}, \quad \phi \ll 1
$$

observe that the dielectric screening by trapped electrons does not resemble, in the far field, a linearized screening. However, on using Eq. (27), Eq. (25) becomes

$$
(r \phi)^{\prime \prime} \approx \frac{2}{\sqrt{\pi}} r \phi^{3 / 2}-r q \delta(\mathbf{r})
$$


so that the classical trapped-electron dielectric screening process approaches, in the far field $(r \gg 1)$, the semiclassical Thomas-Fermi screening (Ashcroft and Mermin $\left.{ }^{4}\right)$ regime!

\section{DISCUSSION}

In this paper, we have given some rigorous results about the properties of the solution of Poisson's equation describing dielectric screening by electrons trapped and/or untrapped in the Coulomb field of the test charge. For the full (untrapped) electrons, the exact screening of the test charge is shown to be weaker (stronger) than linearized screening; this is due to the fact that the linearized screening overestimates (underestimates) the screening of the test charge for the full (untrapped) electrons.

\section{ACKNOWLEDGMENTS}

This work was carried out when the author was a Visiting Research Professor at the Technische Hochschule Darmstadt. The author is very thankful to Professor Peter Mulser for his immense hospitality and valuable discussions. The author also acknowledges with gratitude the helpful criticism of the referee that led to a major sharpening of the discussions. The author is thankful to Dr. David Rollins for his assistance in carrying out the numerical calculations.

${ }^{1}$ N. A. Krall and A. W. Trivelpiece, Principles of Plasma Physics (McGraw-Hill, New York, 1973).

${ }^{2}$ M. A. Lampert and R. S. Crandall, Phys. Rev. A 21, 362 (1980).

${ }^{3}$ S. S. Mak, Plasma Phys. Controlled Fusion 34, 453 (1992).

${ }^{4}$ N. W. Ashcroft and N. D. Mermin, Solid State Physics (Saunders, Philadelphia, 1983). 